\title{
Ablación por radiofrecuencia de haces auriculoventriculares anómalos en niños con taquicardia paroxística supraventricular
}

\author{
Rodrigo Nehgme D. ${ }^{1}$; Alejandro Fajuri N. ${ }^{2}$; Rolando González A. \\ Felipe Heusser R. ${ }^{1}$ Eugenio Marchant D. ${ }^{2}$
}

\section{Radiofrequency catheter ablation of accesory atrioventricular pathways in children with supraventricular tachycardia}

\begin{abstract}
Electrophysiologic studies and a radiofrequency catheter abiotion procedures were perlormed in 21 palients with supravenlricular bachycardia due to accesory atrio venlricular pothways. There wete iwo patients with congenild heat disease. The radiofrequency ablation was successtul in 12/15 (80\%) palienls with left sided anomalous pathways and in $4 / 0,07 \%$ polients with fighi sided pothways ip $>0.05$, chi-square). The procedure was successful in 10/12 (83\%) patients with preexitation (Wolr Parkinson White syndrome) and in $0 / 9(67 \%$ ) with concealed accesory pathways \{ $p>0,05$, chi-square\}. The success iate for the whole series wos $76 \%$ and il was significantly higher [84\%) in palienls withoul congenital hearl disease if $=0,048$. Fisherf. Significart complications included left femoral arlery obstruction wilh leg compartment syndrome and caldiac perforation with tamponade. Radiofrequency catheter ablation is a permanent form of theraphy with a hign success rate for poroxismal supraventricular tachycardia. Complicalions, although infrequent, may be severe, therefore, strict criteria lor polient seleclion are required.
\end{abstract}

(Key words: supraveniticular paroxismal tachyeardia, radidrequency calheler ablalion, preexilation.)

El registro intracavitario de la actividad eléctrica cardiaca o estudio electrofisiológico ha sido por años una herramienta útil en el diagnóstico de los trastornos del ritmo cardiaco y en la evaluación de la terapia farmacológica de los mismos'. En años recientes, la electrofisiología ha penetrado el campo de los procedimientos invasivos terapéuticos con la aparición de técnicas de ablación de focos arritmogénicos o de haces paraespecílicos responsables de reentrada auriculoventricular en las taquicardias paroxísticas supraventriculares (TPSV) ${ }^{2,3}$. La ablación o fulguración por medio de energía de radiofrecuencia es actualmente cl procedimicnto de elccción para el tratamiento definitivo de los pacientes con diagnóstico de TPSV por haces paraespecificos,

1. Laboratorio de Electrofisiologia, Hospital Clínico je la Pontilicia Universidad Carólica de Chilc. Departamento de Pedialría.

2. Laboratorio de Electrofisiología, Hospital Clínico de la Pontificia Universidad Católica de Chile. Departamento de Enfermedades Cardiovasculares. accesorios o anómalos que conectan miocardio auricular y ventricular ${ }^{4-7}$. La experiencia publicada con esta técnica en pacientes pediátricos es escasa ${ }^{8}$. El propósito de esta comunicación es describir nuestra experiencia con el procedimiento, en el tratamicnto de niños con taquicardia paroxística supraventricular frecuente, con mala respuesta al tratamiento farmacológico.

\section{Pacientes y Métodos}

Desde juljo de 1992 a octubre de 1993, 21 pacientes derivados con diagnóstico de TPSV fucron somelidos a estudio electrofisiológico para delerminar las característjcas y ubicar el haz ançmalo responsable de sus taquicardias. La edad promedio de los pacientes fue de 9,7 años y varió enlre 4 a 16 años: 12 eran mujeres. Dos niños sufrían malfornación cardiaca cungênila: uno, de 11 años, enfermedad de Ebsicin no operada y otro, de 10 años, transposición de grandes arterias operada con un procedimiento de tipo Senning. Los pacientes tenían historia de palpitaciones por 8 meses a 9 años, la frecuencia de las crisis variaba desde dos episodios por año hası dos por semana. En todos se había intentado, sin buen éxilo, tratamiento farmacológico ctónico con dos o más drogas antiarrítmicas secuencialmente 
o en forma combinada. El esudio electrofisiológico y la ablación se indicaron debido al Iracaso del tratamiento médico y la frecuencia de las crisus, realizándose en estado posabortivo, bajo sedación con secobarbital $5 \mathrm{mg} \cdot \mathrm{kg}$ oral y morfina $0,1 \mathrm{mg} \cdot \mathrm{kg}$ subcutánea. Las drogas antiarrímicas se habian suspendido por a lo menos cuatro días, para evitar sus efectos electrofisiológicos en el estudio.

Utilizando la técnica percutánea de Seldinger, previa anestesia local con lidocaína al $1 \%$, se pusieron dos vainas calibre 6 french $(F)$ en la vena femoral derecha y una $6 \mathrm{~F}$ en la vena subclavia derecha o la yugular inteme derecha. $A$ través de las vainas femorales se introdujeron catétcress electrodo $6 \mathrm{~F}$ cuadripolares (Mansfield): uno se ubico $\mathrm{en}$ la aurícula derecha para registro de electrograma auricular derecho y estimulación auricular programada; el otro se ubico, a través de la válsula tricúspide, en la posición del haz de His, para cslimulación vertricular programada y registros de electrogramas de aurícula derecha septal, haz de His y ventriculo derecho. A través de la yaina subclavia (o yugular) se introdujo otro caréter clectrodo cuadripolar por el seno coronario para registrar electrogramas de aurícula y ventrículo izquierdos (figura 1). Los registros se realizaron en un poligrafo Mingograf 7 Sicmens-Biema. Los electrogramas fucron filtrados de 25 a $500 \mathrm{hz}$ grabaJos a velocidad variable, desde 10 hasla $200 \mathrm{~mm} / \mathrm{seg}$, con 10 a 40 mvolts de amplificaciôn.

Inicialmente, se realizó estiralación ventricular con frecuencias crecientes, hasta llegat a intervalos $\$ 1-\$ 1$ de 300 milisegundos (ms). Esto pernitió confirmar la existencia de conducción retrógrada ventrísulo-auricular, sugerente de un haz paraespecítico, tanto por la secuencia excéntrica de activación retrógtada ventrículo auricular como por las caracierísticas no decrementales de la misma. Luego se realizó estimulación auricular a frccuencia creciente y auricular programada con cíclos basales S1-S1 de 600,500 y $400 \mathrm{~ms}$ e introducciōn de un extraestímulo $\$ 1-\$ 2$ a intervalos progresivamente menores hasta alcanzar cl periodo refractario auricular. Con este proceder se perseguía inỏu. cir TPSV, definir los períodos refractarios en direccion anterógrada del haz anómalo en pacientes con WolfParkinson-Whitc, como también del nodo auriculoventricular en todos los pacientes y descartar la presencia de doble vía nodal asociada. Esta última condición fue definida como una discontinuidad o "salto" en la condacción auriculoventricular mayor que $100 \mathrm{~ms}$ con la introducción del estímulo extra S1-S2. Se utilizó un cstimolador programable Medtronic 503.

La localixación del haz paraespecífico fue definida según la sccuencia de activación retrógrada ventriculoauricular observada durante la estimulación ventricular o en el transcarso de urá taquicardia paroxística. Asi, por ejemplo, cn casos de haces anómalos laterales izquierdos los electrodos distales del catéter en el seno coronario -que registran electrogramas de aurícula y ventrículo laterales izquicrdosson los primeros en registrar actividad auricular. Se consideró como sitio ideal para la aplicaciốn de radiofrecuenciz la zona del anillo alrioventricular donde se registrasen elecIrogramas ventriculoauriculares fusionados (figura 1) o donde, entre los mencionados electrogramas, se observaran potenciales de haz de Kenl o anúmalo. Altemativamente, en casos de Wolf-Parkinson-White lambién se eligio la zona en que, durante ritmo sinusal, se registrara un potencial de Kent (figura 2).
Una vez deleminada la presencia y localización del haz paracspecífico responsable de la TPSV en cada paciente, se procedió a la aplicación endocárdica de energía de radiofrecuencia (30-50 w). La fuente de encrgia utilizada fue un equipo Osypka Hat $200 \mathrm{~S}$. En casos de haces paraespecíficos locatizados en el anillo auriculoventricular izquierdo se avanzó un catéter de ablación Mansfield 7F desde la arteria femoral derecha, en forma retrógrada a través de la váivula sórtica, cl que se localizó en el endocardio ventricular. ccreano al anillo surículoventricular, por debajo de la inserción de los velos de la valvula mitral. Altemativamente, en tres pacientes con haces izquierdos. la radiofrecuencia fue aplicada en el endocardio auricular cercano al anillo. Para tal cfecto, en dos pacientes el catćter de ablación fue introducido desde la vena femoral derecha hacia la aurícula derccha y luego, a través de un foramen oval permeable, a la

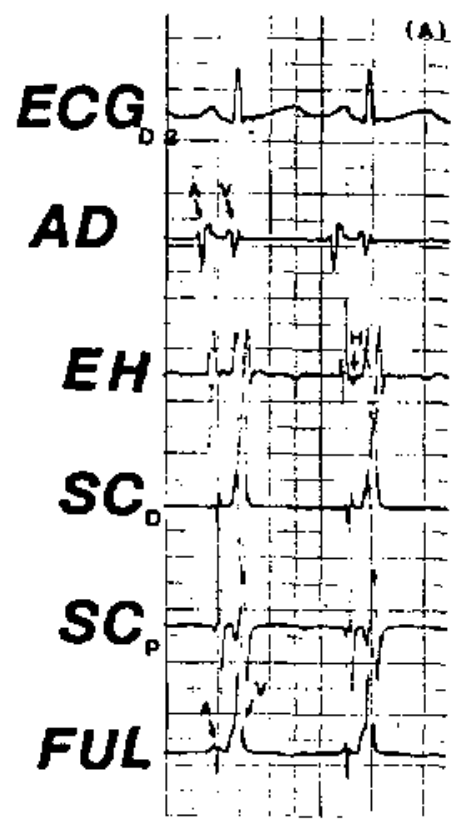

(B)

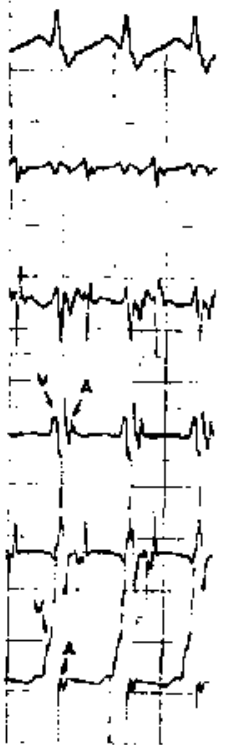

Figura 1. En la colomna A se obseryan los registros obte. nidos durante un EEF realizado en un paciente de 7 años con TPSV. Se observa, de arriba hacia abajo, registro de ECG de superficie (D2) seguido de registros intracavitarios de aurícula derecha ( $A D$ ): His ( $E H$ ) que muestra electrog ramas de auricula septal, haz de His (H) y ventrículo derecho; seno coronario distal (SCD) y proximal (SCP) que muestran electrogramas de aunicula y ventriculo izquierdos. La última línea muestra los registros obtenidos con el catéter de fulguración (FUL), el que se ha ubicado en el anillo A-V izquierdo, en esirecha relación con los elecirodos de SCD. Se puede apreciar la similitud de ambos registros (SCD y FUL). En la columna de la derecha (B) se observa el registro obtenido con los mismos catéteres durante taquicardia. La fusión de los electrogramas $V$ y A observada en el SCD y. más nororiamente, en el registro del catéter de fulguración (FLL) confirma la posición lateral izquierda del HPE. EI silio con potencial $V$-A fusionado es considerado ideal para aplicar RF. 


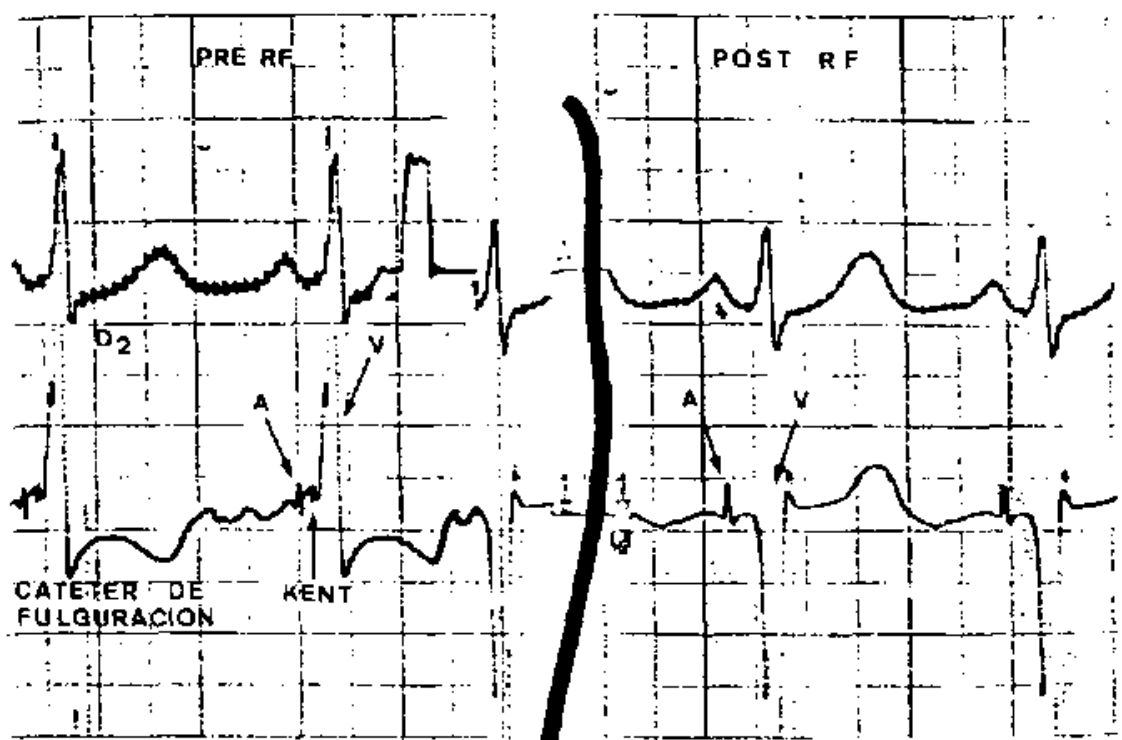

Figura 2. Regisisos obtenidos en una paciente de 6 años con diagnóstico de WPW y TPSV antes (PRE RF) y luego de ablación cxilosa (POST RF). Antes la R PR corto y preexcitación ventricular. El caléter de fulguración registra, entre cl electrograma de auricula (A) y el de ventrículo (V), un potencial pequeño y rápido que corresponde al potencial del HPE o haz de Kent (KENT). Luego de la fulguración desaparece la prexcíación y, ciertamente, el potencial de Kent.

aurícula izquierda. En un paciente de $?$ años, en quien incentos de ablación por vía anterial retrógrada habían fracasado, el catéter de fu]guración se empujó hacia la auricula izquierda por punción transeptal clásica. En el caso de haces paraespecíficos dercchos se hizo avanzar un catéter de ablación Mansfield 7F o EPT 8F desde la vena femoral derecha hacia la aurícula derecha y se aplicó la energía en el endocardio auricular.

En los pacientes con baces izquierdos se uso heparina $100 \mathrm{LT} \cdot \mathrm{kg}$ ev como dosis de carga y lucgo 20 a $50 \mathrm{UI} \cdot \mathrm{kg}$ ev cada una hora, mientras hubiese catéleres en el territorio arterial sistémico. Al aplicar la energía se esperó, para predecir el buen éxilo y continuar la aplicación por 60 a 120 segundos, que desapareciera la preexcitación, se internumpiera la taquicardia o se bloqueara la conducción retrógrada (si se había procedido con escimulación ventricular). Luego de olro período de espera de 30 minutos, se confirmó el bloqueo de la conducçón por cl haz anćmalo mediantc estimulación ventricular. La ausencia de conducción ventriculoauriculat o la conducción decremental nornal por vía nodal se consideró evidencia de buen resultado.

Durante el procedimiento se vigilaron continuamente frecuencis cardiaca, saturación de $\mathrm{O}_{2}$ transcutánea y presión arterial con manguito de insuflación automática. Durante el mismo, se utilizó midazolam $0,1 \mathrm{mg} \cdot \mathrm{kg}$ ev o morfina 0,05 $\mathrm{mg}$ - $\mathrm{kg}$ ev según fuera necesario para mantener al paciente sedado.

Luego del procedimiento, los pacientes fueron obscrva. dos en una uniclad de cuidados intemedios con controles de heınatocrito y radiografía de tórax en las primeras 6 horas, electroca rdiograma cada 8 horas por 24 a 48 horas. Mediante ultrasonografía cardiaca bidimensional y Dopple 24 a 48 horas después del procedimiento se evaluó la función ventricular, se buscó insuficiencia aórtica y mitral, derrame pericárdico o trombos intracavitarios. Los pacientes han sido controlados una semana, un mes y seis meses despues del procedimiento por examen clínico y electrocardiograma. En el control del mes se hizo, además, ulirasonografía.

El análisis estadístico se realizó con un programa 512 Stat View + TM (Apple, MacIatosh). Se le asignó significación estadistica a valores de $p<0,05$ para análisis de chicuadrado y prueba exacta de Fisher.

\section{Resultados}

En todos los pacicntes fue posible inducir taquicardia paroxística por reentrada aurículoventricular ortodrómica con el protocolo mencionado. En un paciente se encontró una vía nodal doble asociada, sin lograr inducirle "ecos" auriculares ni taquicardia por reentrada nodal. La duración de todo el procedimiento variós de 2 a 7 horas. Ел tres pacientes fue necesario repetir el procedimiento y los intentos de ablación: en un niño de 7 años con TPSV de dificil manejo desde el periodo neonatal debida a un haz paraespecífico lateral izquierdo, luego de fracasar dos veces la ablación por vía arterial, se reintentó hacerla por 
vía auricular transeptal; en un paciente de 10 años con transposición de grandes arterias operada con cirugía tipo Senning, debió repetirse por la dificultad en hacer el mapa y la ablación misma y, finalmente, en una paciente de 9 años con Wolf-Parkinson-White y haces múltiples izquicrdos, fue necesario repetir el procedimiento debido a lo prolongado del primero. Por lo tanto, en 21 pacientes se rcalizaron 25 procedimientos electrofisiológicos.

El estudio permitió identificar, en 12 de 21 pacientes $(57 \%)$, el sindrome de Wolf-ParkinsonWhite. En ellos fue posible, por lo tanto, demostrar conducción anterógrada y retrógrada a través del haz paraespecífico. Los restantes nueve pacientes $(43 \%)$ terían un haz oculto o s6lo con conducción retrograda. En estos últimos nif̃os no se registraba preexcitación ventricular en el electrocardiograma de superficic, basal o bajo estimulación auricular rápida.

En 15 (71\%) pacientes los haces anómalos eran izquierdos y en seis (29\%) derechos. El paciente con anomalía de Ebstein tenia múltiples haces derechos y el niño con trasposición operada uno izquierdo.

Se obtuvo buen resultado en 10 de $12(83 \%)$ pacientes con preexcitación y en 6 de $9(67 \%)$ niños con haces ocultos ( $p=0,375$, chi-cuadrado), en 12 de $15(80 \%)$ casos con haces izquierdos y 4 de los $6(67 \%)$ portadores de vías anómalas derechas $(p=0,517$, chi-cuadrado). Tampoco se observó una diferencia significativa en el éxito obtenido al comparar a los pacientes mayores y menores de diez años de edad $(p=0,696$, chicuadrado). En el total de la experiencia, la ablación dio buenos resultados cn 16 de 21 pacicntes (76\%). No se obtuvo éxito en ninguno de los niños con cardiopatia congénita. Excluyendo a estos últimos, el éxito fuc significativamente mayor de $84 \%$ ( $p=0,048$ Fisher). En el pcríodo de observación inmediatamente después del procedimiento, una paciente de 16 años desarrolló neumotorax apical derecho mínimo, asintomático y que no requirió punción ni drenaje. El paciente de 11 años con enfermedad de Ebstein sufrió parálisis frénica derecha transitoria. Una paciente de 5 años presentó obstrucción transitoria de la arteria femcral derecha, sin compromiso de la vitalidad de la extremidad, de la que se recuperó totalmente con heparinización sistémica por 24 horas. En un paciente de 7 años con Wolf-Parkinson-White por haz lateral izquierdo, TPSV recurrente de difícil manejo médico desde el periodo nconatal, sucedieron las dos complicaciones más graves de nuestra seric: perforación y taponamiento cardiacos en relación a un intento de ablación por via transeptal y, en un procedimiento posterior, obstrucción arterial con sindrome de compartimiento que requirió trombectomía quirúrgica, fasciotomía y debridación de tejido muscular necrótico. En el control ultrasonográfico realizado un mes después del procedimiento se pesquisó, en un paciente, derrame pericárdico posterior, pequeño (1 cm en el eje corto paraestemaI), asintomático, que desapareció al cabo de una semana de tratamiento con ibuprofeno y se interpretó como secundario a irritación pericárdica (sindrome de Dressler).

A lo largo del seguimicnto (media 5,9, márgenes 1 a 13 meses) no se han registrado crisis de taquicardia ni reaparición de la preexitación en los pacientes en quienes la ablación dio buenos resultados inicialmente. No hubo mottalidad en nuestra scrie.

\section{Comentario}

El tratamiento definitivo de los pacientes con TPS $V^{\prime}$ por haces paracspecíficos consiste en la sección quirúrgica o mediante catéteres de dichas vías anómalas de conducción auriculoventricular. La ablación por corricnte continua, alterna o radiofrecuencia a través de catéteres, ha desplazado a la cirugía en el tratamiento de estas arritmias $^{2.3}$. La corricnte continua ( 25 a 300 joules) actua por barourauma, pues produce una onda expansiva por formación súbita endocavitaria de guses. A pesar de su eficiencia, los inconvenientes del barotrauma, descritos en adultos y niños, pueden scr importantes $\mathrm{c}$ incluyen derrame pericárdico, perforación con taponamicnto, arritmias auriculares y ventriculares, fibrilación ventricular, bloqueo auriculoventricular, espasmo coronario y la necesidad de emplear anestesia general ${ }^{2,3}$. Por otro lado, la ablacjón por radiofrecuencia sólo emplea el aumento de temperatura localizado en el extremo del catéter correspondiente, por cso las lesiones tisulares son más pequeñas $\left(0,2 \mathrm{~cm}^{2}\right)$ y menos profundas, por consiguiente el ricsgo de efectos adversos es tambjén monor. Esta técnica ha resultado, además, muy efectiva, con alrededor de $90 \%$ de buenos éxitos en adultos y niños ${ }^{4-8}$. 
La experiencia que se comenta mucstra que es posible realizar estudios electrofisiológicos diagnósticos y terapéuticos en nuestro medio y corresponde a la primera publicación pediátrica nacional sobre electrofisiología y ablación por radiofrecuencia. El éxito obtenido en la ablación por radiofrecuencia de haces paracspecíficos confirma que el procedimiento es una forma adecuada y definitiva de tratamicnto para pacientes con taquicardia paroxística supraventricular, independientemente de la localización de la vía anómala o de su capacidad de conducir en una o dos direcciones (oculta o con preexitación). A pesar las diferencias registradas en el éxito del procedimicnto entre pacientes con y sin malformaciones congénitas del corazón en esta serie, el reducido número de estos últimos obliga a hablar sólo de una tendencia al fracaso en ellos. Los resultados favorables en los demás pacientes son semejantes a los de otras experiencias en adultos y niños ${ }^{4-8}$.

Las complicaciones observadas en esta serie son del mismo orden que en otras ${ }^{4,5,7}$. No hemos detectado en estos niños la aparición de insuficiencia aórtica o mitral, que ha inducido a algunos a realizar el procedimiento por via transeptal ${ }^{9}$. Sin embargo, a pesar de la baja frecuencia $y$ significado de las complicaciones en nuestros casos, éstas pueden ser graves otras veces, lo que exige rigurosidad cn la selección de los pacientes a estudiar. Los candidatos al procedimiento deberían ser niños con TPSV de larga evolución y crisis frecuentes que causen múltiples consultas de urgencia, hospitalizaciones, trastornos psicosociales y miocardiopatia; pacientes refractarios al tratamicnto con medicamentos de primera línea (digital, propanolol) o que sulren efectos adversos con el tratamiento farmacológico crónico.

\section{Resumen}

En 21 pacientes (edad promedio 9,7, márgenes 4 a 16 años) con taquicardias paroxísticas supraventriculares por haces paracspecíficos, se realizaron estudios electrofisiológicos y ablación de los haces por radjofrecuencia. Dos pacientes tenían malformaciones congénitas del corazón. La ablación se consiguió en 12/15 haces anómalos izquierdos (80\%) y en $4 / 6$ derechos $(67 \%)$, en 10/12 pacientes con preexcitación
(83\%) y $6 / 9$ haces ocultos (67\%) (diferencias no significativas). El buen éxito se obtuvo en $76 \%$ (16/21) del tolal de pacientes, pero éste aumentó a $84 \%$ ( $\mathrm{p}=0,048$ Fisher $)$ después de eliminar los pacientes con malformaciones congénitas. Las complicaciones observadas incluyeron obstrucción arterial con sindrome de compartimicnto y perforación con taponamiento (un caso de cada una). La ablación por radiofrccuencia es un tratamiento adecuado y definitivo en niños con taquicardia paroxística supraventricular, independientemente de la localización de la váa anómala y la presencia de preexitación, pero puede fracasar en casos de malformaciones cardíacas. Las complicaciones de importancia son poco frecuentes, pero pueden llegar a ser graves, lo que exige una rigurosa selección de los pacientes a estudiar.

(Palabras clave: taquicardia paroxística supraventricular, haces paraespecíficos, preexitación, estudio electrofisiológico, resección endocavitaria, catétcr electrodo, radiofrecuencia.)

\section{Referencias}

1. Subcommittee to asses clinical intracardiac electrophy. siologic studies: Guidelines for clinical intracardiac electrophysiologic studies. A report of the American College of Cardiology/American Ileart Association task force on assessment of diagnostic and therapeutic eardiovascular procedures. J. Am. Coll Cardiol. 1989; 14: $1827-42$

2. Scheiman M.: Catheter Ablation. Present role and projected impacl on healt care for patiens with cardiac arrhythmias. Circuldion, 1991: 83: 1489-1498.

3. Scheinman $M$, Laks $M$, DiMarco J, Piumb V.: Current role of catheter ablative proccdures in patients with car diac arrhythmias Circulalion, 1991; 83: 2146-2153.

4. Jackman W, Zunzhang W. Friday K. Moulton K, Becknan $K$, McCleliand $J_{s}$ Ruidale $N$, Haziul $J$, Prior M, Margolis $P$ : Calame J, Overholt $F_{n}$ Lazzara R.: Catheter ablation of accesory atrioventricular pathways (Wolff-ParkinsonWhile Syndrome) by radiof requency current. N Engl J Med 1991; 324: 1605-1611.

5. Calkins H, Sousa J, Et-Atassi R, Rosenheck S, DeBuitler $M, K o w W, K a d i s h$ A, Langberg J, Morady $F .:$ Diagnosis and cure of the Wolff-Parkinson-White Syndrome or paroxismal supraventricular tachycardja during a single electrophysiologic test. N Engl J Mcd. 1991; 324: 1612 1618.

6. DeBuitter M, Sousa J, Bolling S, El-Atassi R, Calkins H, Langberg $J$, Kou W, Morady $F .:$ Reduclion in medical care cost associated with radiof requency cathecer ablalion of accesory pathways. Am J Cardiol., 1991: 68: 1656-1661.

7. Calkins H, Kim Y, Schmaliz S, Sausa $J, E l$-Atassi $R$, Leon A, Kadish A, Langberg $J$, Morady $F_{\text {.: Electrogram }}$ 
criteria for identification of appropriate target sites for radiofrequency catheter ablation of accesory atrioventricular connecions. Circulation, 1992; 85: 565 573.

8. Van Hare G, Lesh M, Scheinman M, Langberg J.: Percutaneous radiofrequency catheter ablation for supra- ventricular arrhythmias in children. J. Am. Coll. Cardiol. $1991 ; 17: 1613-1620$.

9. Minich $L$. Snider $R$, MacDonald $D .:$ Doppler detcction of valvular tegurgitation after radiofrequency ablation of accesory connection. Am. J. Cardiol., 1992; 70: $116-117$

\section{AVISO A LOS AUTORES}

Por acuerdo del Comité Editorial, la Revista Chilena de Pediatría devolverá sin tramitar todos los trabajos que no den estricto cumplimiento al Reglamento de Publicaciones y a las Instrucciones de los Autores que se editan en cada número de la Revista. 